\title{
COMPARAÇÃO ENTRE A UTILIZAÇÃO DE EXERCITADORES E JOGOS DE VIDEOGAME NO FORTALECIMENTO DA MÃO
}

\author{
B. G. KIMURA*, N. N. ZAGO**, L. F. R. M. FERNANDES***
}

\author{
*Graduando em Fisioterapia, Universidade Federal do Triângulo Mineiro, Uberaba, Brasil \\ **Discente do Programa de Pós-Graduação em Educação Física, Universidade Federal do Triângulo \\ Mineiro, Uberaba, Brasil \\ ***Docente do Departamento de Fisioterapia Aplicada, Universidade Federal do Triângulo Mineiro, \\ Uberaba, Brasil \\ e-mail: brunogkimura@gmail.com
}

Resumo: A reabilitação com o uso do videogame fornece um feedback visual e auditivo e proporciona inúmeros benefícios na recuperação da capacidade física e cognitiva, além de possibilitar uma nova experiência, diferente d a terapia convencional. Este estudo teve como objetivo comparar o efeito do videogame, como ferramenta terapêutica, em relação a um programa de fortalecimento convencional para os músculos da preensão e das pinças. Foram selecionadas por conveniência 12 mulheres, com idade média de 22 $( \pm 2,0)$ anos, sendo todas com ausência de doenças neuromusculares e musculoesqueléticas. Para realização desse estudo foi utilizado o kit E-link d a marca Biometrics ${ }^{\circledR}$ modelo H500 - Hand kit, o software de avaliação para os membros superiores ( $E W S-U p p e r$ Extremity Evaluation Software) e material de mecanoterapia (kit exercitador da marca Digiflex $®$ ). As voluntárias foram divididas em dois grupos: grupo exercitador (GE) e grupo videogame (GV), ambos com 6 mulheres. Para o fortalecimento foi utilizado o protocolo Oxford. Os dois grupos realizaram fortalecimento da preensão grossa, pinças polpa-polpa, polpa-lateral, polpa-trípode. $\mathrm{Na}$ análise intragrupos, para o GE foi encontrado diferença significativa no aumento da força pré e pós fortalecimento para as pinças polpa-polpa, polpa-lateral; para o G V foi encontrado diferença significativa para força de preensão palmar. $\mathrm{Na}$ análise intergrupos foram encontrados maiores ganhos da força no GV em relação ao GE para todas as forças, porém esse ganho não foi significativo. Este estudo demonstrou que o grupo que realizou o fortalecimento com o videogame obteve um ganho maior de força de preensão e pinça, quando comparado ao GE.

Palavras-chave: Treinamento de Resistência, Força Muscular, Força da Mão, Força de Pinça.

\footnotetext{
Abstract: The rehabilitation using videogame supplies an auditory and visual feedback and provides many benefits in the recovery of physical capacity and cognitive ability, besides to enable a new experience, different from conventional therapy. This study aimed to compare the effect of the videogame as a therapeutic tool in relation to a conventional therapy program for handgrip and pinch muscles. For convenience were selected 12 women,
}

with mean age of $22( \pm 2,0)$ years, all with the absence of neuromuscular and musculoskeletal diseases. To performer this study was used the Kit E-link brand Biometrics ${ }^{\circledR}$ model H500 - Hand kit, the evaluation software for the upper extremity (EWS - Upper Extremity Evaluation Software) and mechano therapy material (Kit exerciser brand Digiflex $囚)$. The volunteers were dividing in two groups: exerciser group (EG) and videogame group $(V G)$, both with 6 women. All used the Oxfordstrengthening protocol. The booth groups performed handgrip, pinch tip-to-tip, key and three-jaw strengthening. In the intragroup analysis for EG were founded significant difference in the increase of pre and post strength to grip tip-to-tip and key; and for the VG were founded significant difference in the handgrip strength. In the intergroup analysis were founded a better gain of strength in the VG when compared with EG for all forces, but this gain were not significant. This study showed that the group whoperformed the videogame strengthening protocol obtained a higher handgrip and pinch force when compared to $E G$.

Keywords: Resistance Training, Muscle Strength, Hand Strength, Pinch Strength.

\section{Introdução}

Atualmente a utilização de jogos para a reabilitação tornou-se uma forma promissora para o estímulo, adesão e incentivo na realização de exercícios $[1,2]$. O ambiente virtual permite a interação do indivíduo com o tratamento, criando sensações de uma realidade diferente d a terapia convencional [3] e, promovendo assim, a percepção da melhora na função e força de seus membros [4].

$\mathrm{O}$ videogame além de ser u m instrumento lúdico, fornece um feedback visual e auditivo e proporciona diversos benefícios na recuperação da incapacidade física e/ou cognitiva [5]. Videogames como o Nintendo Wii ${ }^{\circledR}$ e o Xbox com Kinect ${ }^{\circledR}$ vêm sendo utilizados como recursos para tratamento fisioterapêutico, entretanto estes videogames comerciais são mais específicos para realização de movimentos globais e não visam o fortalecimento muscular.

Para a reabilitação da mão existe no mercado o Kit E- Link da marca Biometrics ${ }^{\circledR}$, que tem jogos de videogame 
acoplados aos dinamômetros de preensão e pinça que funcionam como biofeedback para o ganho de força. A associação do videogame na rotina fornece ao paciente uma maior interação com a terapia e proporciona a sensação de experimentar uma realidade diferente da terapia convencional.

Buscando auxiliar o fisioterapeuta na escolha de protocolos adequados para fortalecimento da musculatura da mão e avaliar se o videogame, além de aumentar o interesse e a motivação dos participantes, também leva ao ganho de força muscular, esse projeto tem como objetivo avaliar a eficácia do videogame, como ferramenta terapêutica no fortalecimento dos músculos da preensão e das pinças em mulheres.

\section{Materiais e métodos}

Esta pesquisa caracteriza-se por s e r u m estudo piloto, composto por 12 mulheres jovens, com idade média de $22( \pm 2,0)$ anos. Os critérios de inclusão foram: a) sexo feminino, b) idade entre 18 e 30 anos, c) que não realizassem exercícios para os membros superiores, e d) ausência de doenças neuromusculares e musculoesqueléticas nos membros superiores.

Todas as voluntárias foram informadas sobre os objetivos e procedimentos da pesquisa e assinaram um Termo de Consentimento Livre e Esclarecido, conforme resolução nº66/12 do Conselho Nacional de Saúde

(CNS). A pesquisa foi aprovada pelo Comitê de Ética em Pesquisa da Universidade Federal do Triângulo Mineiro, com parecer 2416.

Para o desenvolvimento da pesquisa foram utilizados:

a) o Kit E-link da marca Biometrics ${ }^{\circledR}$ modelo H500 Hand kit composto por goniômetros e dinamômetros de preensão e de pinça; b) software de avaliação para os membros superiores (EWS - Upper Extremity Evaluation Software) e c) material de mecanoterapia (kit exercitador da marca Digiflex $\left.{ }^{\circledR}\right)$.

As voluntárias que concordaram e m participar da pesquisa foram randomizadas aleatoriamente e $\mathrm{m}$ dois grupos: grupo exercitador (GE), e grupo videogame (GV). O GE foi composto por 6 mulheres submetidas ao fortalecimento c o mo protocolo de Oxford (carga decrescente) por meio do exercitador de mão e dedos da marca Digiflex ${ }^{\circledR}$. Enquanto o $\mathrm{GV}$ foi formado por 6 mulheres submetidas ao mesmo protocolo de fortalecimento utilizando o videogame E-Link da marca Biometrics ${ }^{\circledR}$.

Inicialmente foi realizada uma avaliação fisioterapêutica contendo: dados pessoais, medidas de força muscular instrumental máxima e teste de 10 repetições máximas (10RM) - para cálculo das cargas da preensão e pinças (polpa-polpa, polpa-lateral, polpatrípode). Os valores da força muscular instrumental (preensão e pinças) foram avaliados no primeiro dia e ao término do programa de fortalecimento.

$\mathrm{Na}$ avaliação da força instrumental de preensão e das pinças foram realizadas 3 repetições nos dois membros alternadamente e calculadas a média das três medidas das forças isométricas máximas. Durante esta avaliação as voluntárias permaneceram sentadas, com o cotovelo fletido a $90^{\circ}$, antebraço e punho em neutro, segundo recomendação da Sociedade Americana dos Terapeutas da Mão (ASHT) [6,7]. A posição da abertura da mão ou empunhadura do dinamômetro variam do posicionamento um até cinco. Em um estudo realizado por Fernandes et al. (2011) a maior força encontradas para os homens foi na posição 3 e, para as mulheres, na posição 2. Sendo assim, nesse estudo foi utilizada a empunhadura do dinamômetro na posição 2 para esse grupo de mulheres avaliadas [8].

O treinamento de ambos os grupos foi realizado no membro que apresentou a menor força de preensão e pinças, e seguindo os regimes isotônicos da técnica de Oxford, sendo a primeira série com $100 \%$, a segunda série com $75 \%$ e a terceira série com $50 \%$ da carga do 10RM. Foram realizadas 3 séries de 10 repetições mantendo a isometria de 5 segundos e o repouso de 5 segundos em cada repetição, totalizando 2 minutos de treinamento e repouso de 2 minutos entre as séries. Esse protocolo foi adaptado ao Protocolo de Oxford desenvolvidos por Zinovieff (1951).

Os dois grupos realizaram fortalecimento da preensão grossa, pinça polpa-polpa, pinça polpa-lateral e pinça polpa-trípode, como demonstrado na Figura 1. Foram realizados alongamentos para o $\mathrm{s}$ músculos flexores e extensores do punho e dedos ao início e ao termino dos protocolos.

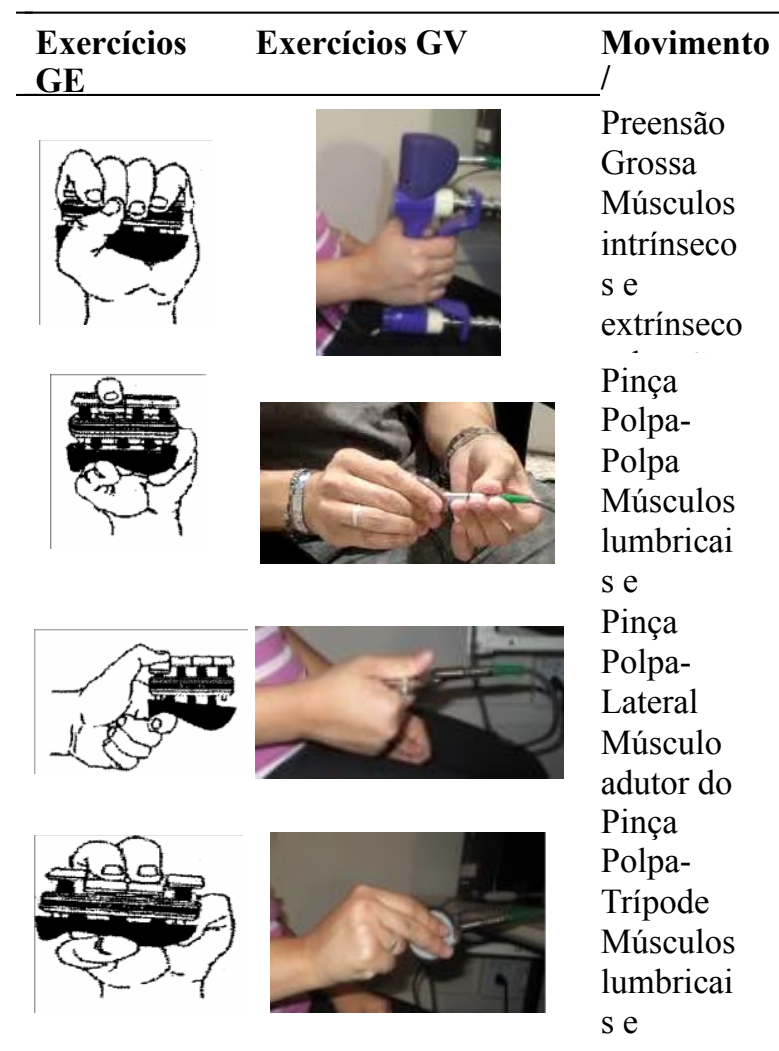

Figura 1: Posições e músculos dos protocolos de fortalecimentos.

Os jogos selecionados para o fortalecimento do $\mathrm{GV}$ foram 1) Monkeys - para preensão grossa, 2) Hit Walls- 
para pinça polpa-polpa, 3) Package- para pinça polpalateral, 4) Skateboard - para pinça polpa-trípode.

Nessa fase foram mantidas 2 sessões semanais, com duração de 40 minutos, totalizando 18 sessões. Para análise dos dados foram considerados os valores do início e após as 18 sessões de fortalecimento.

Análise Estatística - Os dados foram submetidos a análise descritiva e inferencial, c o m nível de significância de 5\%. Na análise estatística descritiva, utilizou-se a média e o desvio padrão. $\mathrm{N}$ a estatística inferencial, para verificação da normalidade, utilizou-se o teste de Shapiro-Wilk. Para a análise intragrupos, o teste " $t$ " de Student pareado, e para análise intergrupos foi utilizado o teste " $\mathrm{t}$ " de Student independente. Os dados foram processados por meio do programa Statistical Package for the Social Sciences (SPSS ${ }^{\circ}$ ) versão 20 .

\section{Resultados}

$\mathrm{Na}$ análise intragrupos, para o GE observou-se diferença significativa no aumento de força pré e pós fortalecimento para as pinças polpa-polpa, polpa-lateral e um aumento não significativo para as forças de preensão palmar e pinça polpa-trípode como demonstrado na Figura 2.

\section{Grupo Exercitador}

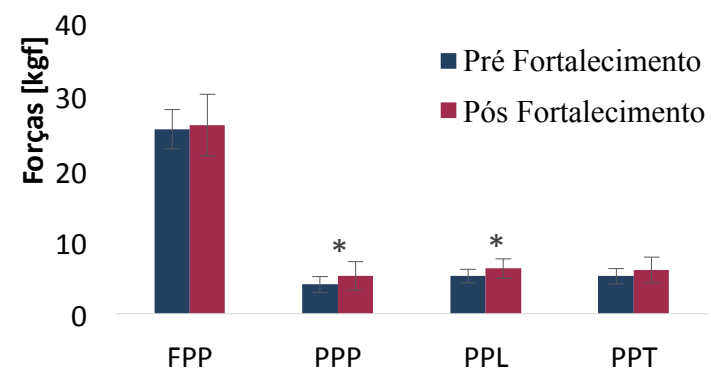

Figura 2: Representação gráfica das forças de preensão palmar (FPP), pinça polpa-polpa (PPP); pinça polpalateral (PPL), pinça polpa-trípode (PPT) pré e pós fortalecimento do GE.

*Nível de significância $\mathrm{p}<0,05$.

E para o GV houve diferença significativa no ganho de força pré e pós fortalecimento para a força de preensão palmar e um aumento não significativo para as forças de pinças como demonstrado na Figura 3.

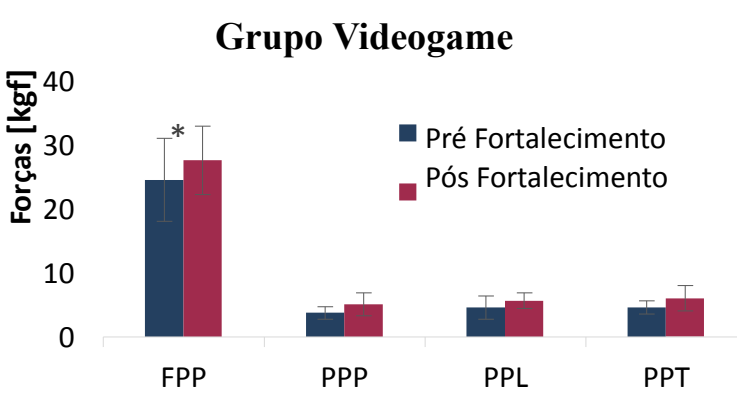

Figura 3: Representação gráfica das forças de preensão palmar (FPP), pinça polpa-polpa (PPP); pinça polpa- lateral (PPL), pinça polpa-trípode (PPT) pré e pós fortalecimento do GV.

*Nível de significância $\mathrm{p}<0,05$.

$\mathrm{Na}$ análise intergrupos foram encontrados maiores ganhos da força no GV em relação ao GE para todas as forças, porém esse ganho não foi significativo (Tabela 1).

Tabela 1: Médias e desvios padrão das diferenças das forças pré e pós fortalecimento.

\begin{tabular}{clcc}
\hline & Grupo & $\begin{array}{l}\text { Média (DP) } \\
\text { da } \\
\text { diferença }\end{array}$ & $\begin{array}{c}\text { p } \\
\text { value }\end{array}$ \\
\hline FPP & GE & $0,58( \pm 1,84$ & 0,109 \\
& GV & $3,03( \pm 2,86$ & \\
PPP & GE & $1,20( \pm 0,93$ & 0,852 \\
& GV & $1,35( \pm 1,77$ & \\
PPL & GE & $1,01( \pm 0,44$ & 0,912 \\
& GV & $1,08( \pm 1,36$ & \\
PPT & GE & $0,86( \pm 1,29$ & 0,484 \\
& GV & $\underline{1,43( \pm 1,40)}$ &
\end{tabular}

$\mathrm{FPP}=$ força de preensão palmar; PPP = pinça polpa-polpa; $\mathrm{PPL}=$ pinça polpa lateral; $\mathrm{PPT}=$ pinça polpa-trípode; $\mathrm{GE}$ = grupo exercitador; $\mathrm{GV}=$ grupo videogame; Diff = diferença das forças pré e pós fortalecimento; DP = desvio padrão.

*Nível de significância $\mathrm{p}<0,05$.

\section{Discussão}

Este estudo teve como objetivo comparar o efeito do videogame, como ferramenta terapêutica, em relação a um programa de fortalecimento convencional para os músculos da preensão e das pinças.

A hipótese do estudo foi verificar se o treinamento com o videogame era mais eficaz no ganho da força dos músculos da mão em relação ao exercitdor convencional, pois o videogame permite a realiação de uma reabilitação mais lúdica.

O protocolo de Oxford foi escolhido para esse estudo, por demonstrar maior eficácia para os músculos da mão por ter como princípio, a redução da resistência a medida que o músculo fadiga e por apresentar melhores resultados quando comparado ao protocolo Delorme [10]. $\mathrm{Na}$ análise intragrupo, tanto o GE quanto o GV apresentaram aumento após fortalecimento para todas as forças. Para o GE os resultados foram significativos para as forças de pinça (polpa-polpa e polpa-lateral). E para o GV o aumento foi significativo apenas para a preensão palmar.

Hutzler et al. (2013) em seu estudo, avaliou as forças de preensão e pinças por meio do Kit E-Link da

Biometrics ${ }^{\circledR}$, pré e pós intervenção com exercícios resistidos (halteres, exercitador, faixa elástica). Nesse estudo, a utilização dos exercitadores, para

fortalecimento dos músculos intrínsecos da mão, teve um resultado positivo no aumento das forças de pinças, corroborando com os resultados encontrados para o GE.

Em relação ao $\mathrm{GV}$, o uso de ambientes virtuais pode ser de grande valia para o processo de reabilitação, 
principalmente quando e la é feita utilizando jogos específicos que respeitem as limitações de cada indivíduo, oferendo ao fisioterapeuta ferramentas de análise da evolução e resultados de tratamento. Os resultados encontrados nesse estudo para o GV condizem com estudos anteriores que demonstraram efeitos positivos da prática em ambiente virtual no aumento da força para o membro superiores [12-14].

Apesar do aumento significativo de algumas forças em cada grupo, os resultados do ganho de força (representandos pela diferença entre o pós e pré fortalecimento) do presente estudo desmonstraram que não houve diferença significativa na análise intergrupo, após a realização do protocolo proposto. No entanto, o ganho de força foi maior para o GV.

Resultados positivos com o uso do videogame $E$ Link foram apresentados por Rostami et al. (2015) que avaliou o efeito da prática em ambiente virtual na função do membro superior de crianças com paralisia cerebral espástica utilizando o videogame E-Link. E comprovaram que a utilização do ambiente virtual apresenta potencial para uma intervenção eficaz no ganho da função da mão, que incluem a velocidade, a força, a amplitude de movimento e a acurácia exigida nos jogos.

\section{Conclusão}

Este estudo demonstrou que o grupo que realizou o fortalecimento com o videogame obteve um ganho maior de força de preensão e pinça, quando comparado ao grupo que realizou fortalecimento com exercitador.

Pretendemos dar continuidade ao estudo com um maior número de voluntários afim de verificar se existe diferença entre os protocolos.

\section{Agradecimentos}

Os autores agradecem ao LABCOM por ceder espaço e equipamentos, a Coordenação de Aperfeiçoamento de Pessoal de Nível Superior (CAPES) e a Fundação de Amparo à Pesquisa de Minas Gerais (FAPEMIG) pelo apoio financeiro e as voluntárias que aceitaram participar, tornando possível a realização desse trabalho.

\section{Referências}

[1] CAMEIRÃO M, BADIA S, VERSCHURE P. Virtual reality based upper extremity rehabilitation following stroke: A review. J Cyber Ther Rehabil. 2008;1(1):63-74.

[2] CHANG Y-J, CHEN S-F, HUANG J-D. A Kinectbased system for physical rehabilitation: A pilot study for young adults with motor disabilities. Res Dev Disabil. 2011 Nov;32(6):2566-70.

[3] ALBUQUERQUE EC, SCALABRIN EE. The Use of the Computer in Neuropsychological Reabilitation Programs. Psicol Argum.
2007;25(50):269-275.

[4] DE GRANDE AAB, GALVÃO FR DE O, GONDIM CA. Reabilitação virtual através do videogame: relato de caso no tratamento de um paciente com lesão alta dos nervos mediano e ulnar. Acta Fisiatr. 2011;18(3):157-62.

[5] BATISTA J, WIBELINGER L, MARCHI A, SCHNEIDER R, PASQUALOTTI A. Reabilitação de idosos com alterações cognitivas através do videogame Nintendo Wii. RBCEH. 2012 maioago;9(2):292-9.

[6] FESS EE. Grip Strength. 2nd ed. Chicago: American Society of Hand Therapists; 1992.

[7] FESS EE, MORAN CA. Clinical assessment recommendations. Philadelphia: American Society of Hand Therapists; 1981.

[8] FERNANDES LFRM, BERTONCELLO D, PINHEIRO NM, DRUMOND LC. Correlações entre força de preensão manual e variáveis antropométricas da mão de jovens adultos. Fisioter E Pesqui. 2011;18(2):151-156.

[9] ZINOVIEFF A. Heavy-resistance exercises the "Oxford technique". The British journal of physical medicine: including its application to industry. 1951;14(6):129-32.

[10] FERNANDES L, ARAÚJO MS, MATHEUS JPC, MEDALHA CC, SHIMANO AC, PEREIRA GA. Comparação de dois protocolos de fortalecimento para preensão palmar. Rev Bras Fisioter. 2003;7(1):17-23.

[11] HUTZLER Y, RODRÍGUEZ BL, LAIZ NM, DÍEZ I, BARAK $S$. The effects of an exercise training program on hand and wrist strength, and function, and activities of daily living, in adults with severe Cerebral Palsy. Res Dev Disabil. 2013 Dec;34(12):4343-54.

[12] JANG SH, YOU SH, HALLETT M, CHO YW, P A R K C - M , C H O S-H, et al. Cortical Reorganization and Associated Functional Motor Recovery After Virtual Reality in Patients With Chronic Stroke: An Experimenter-Blind Preliminary Study. Arch Phys Med Rehabil. 2005 Nov;86(11):2218-23.

[13] ROSTAMI H, NEJAD S, ARASTOO A. Effects of Practice in Virtual Environment on Upper Limb Function of Children with Spastic Hemiparetic Cerebral Palsy. Iran Neurology. 2015.

[14] SVEISTRUP H. Motor rehabilitation using virtual reality. J Neuroengineering Rehabil. 2004;1(1):1. 